\title{
The Performance based Evaluation of Models and Routing Protocols for Indian Automotive Networks
}

\author{
Linesh Raja \\ Research Scholar (SCSS) \\ Jaipur National University, \\ Jaipur, Rajasthan, India
}

\author{
V.S. Dhaka \\ Professor (SOET) \\ Jaipur National University, \\ Jaipur, Rajasthan, India
}

\author{
R.C. Poonia \\ Assistant Professor (AllT) \\ Amity University \\ Jaipur, Rajasthan, India
}

\begin{abstract}
In this paper we evaluate the performance of mobility models, radio propagation models and routing protocols for intelligent transport system in Indian Automotive Networks. The evaluation helps us in selecting the appropriate models and protocols for the urban and rural scenario. This paper also helps us to identify the suitable propagation model, WAVE IEEE standards, wireless channels and the type of flow required for the Indian Automotive Networks.
\end{abstract}

\section{Keywords}

WAVE, Ad-hoc, IEEE, ITS, VANET

\section{INTRODUCTION}

The Vehicular Ad hoc Networks (VANETs) is a type of adhoc network where the vehicles act as node [1] [2]. In VANET vehicles are highly mobile and need to transmit packets to each other using the wireless channel. The vehicles must configure with wireless transceivers and computerized modules that help the vehicles to act as network nodes [3].

VANET is related to Mobile Ad hoc Network (MANET) in term of its basic principle, but it does not rely on fixed infrastructure for communication and data dissemination; however permanent network nodes in the form of roadside units can be useful in some circumstances. VANET also shares a few common MANET characteristics such as selforganization and self-management, short radio transmission range and limited bandwidth [3].

VANET can be observed as component of the Intelligent Transportation Systems (ITS). In ITS, vehicles communicate with each other via Inter-Vehicle Communication (IVC) as well as with roadside base stations via Roadside-to-Vehicle communication (RVC) for safety purpose. Inter Vehicle Communication i.e. car-to-car communication is useful for enhancing traffic safety, comfort driving and in-car entertainment [2] [4].

As this paper helps in the appropriate selection of the mobility models, radio propagation model and routing protocols for the realistic evaluation of the Indian automotive networks. This is one of the challenges faced by the researchers, when they are working on VANETs. Especially in Indian automotive networks, this is a great concern. So selecting the suitable mobility model i.e. macro and micro mobility etc., radio propagation model i.e. TwoRayGround, Nakagami etc. will boost the performance of the VANETs. At the same time selecting the appropriate routing protocol i.e. AODV, DSR, TORA etc. for the Indian automotive networks is also a concern for getting better result.

The organization of this paper is as follows. The second and third section of this paper offers the brief study of the mobility and propagation models respectively. To achieve the target various mobility and propagation model is be studied. Selecting the suitable Models for VANET is a challenge. The section four of this paper elaborates the various routing protocols. Section five presents the analysis of the above sections. The last section provides the conclusion of this paper.

\section{MOBILITY MODELS}

Mobility is a major component for providing quality to the realistic simulation. It is an important issue for Indian automotive network as they grow and their transportation facilities become congested. Large number of techniques is available to improve traffic flow to minor physical changes, such as adding a lane by elimination of parking. However the problem lies in evaluating the performance of these techniques [5]. The need is to evaluate traffic performance in a network under various traffic and geometric configuration. There are two aspects of vehicular mobility model which may be considered for VANET. They are:

- Multilayer description of vehicular mobility patterns: A critical aspect in a simulation study of VANET is the need for a mobility model that would reflect the real behavior of vehicular traffic, as vehicular mobility considerably impacts the networking shape of VANET [6]. Vehicular mobility is usually considered at various modeling layers i.e. Trip, Path and Flow Modeling.

- Bi-directional interaction between traffic and network simulators: A major distinctive fact of vehicular communication applications is to benefit from the messages exchanged between cars to alter traffic, either for safety purposes with advanced safety messages, or for traffic efficiency in order to avoid traffic jams. In both cases, a strong interaction must be defined between the network protocol and the vehicular mobility.

There exist various models reproducing mobility of nodes [7]. Stochastic mobility models, like Random Way-Point or models with constrained movement (Manhattan) are not able to reproduce the realistic traffic mobility [8]. It is shown in fig. 1 and 2 respectively.

$$
\begin{aligned}
& \text { 7i }
\end{aligned}
$$

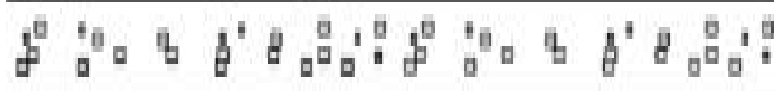

Fig 1: Freeway Mobility Model 


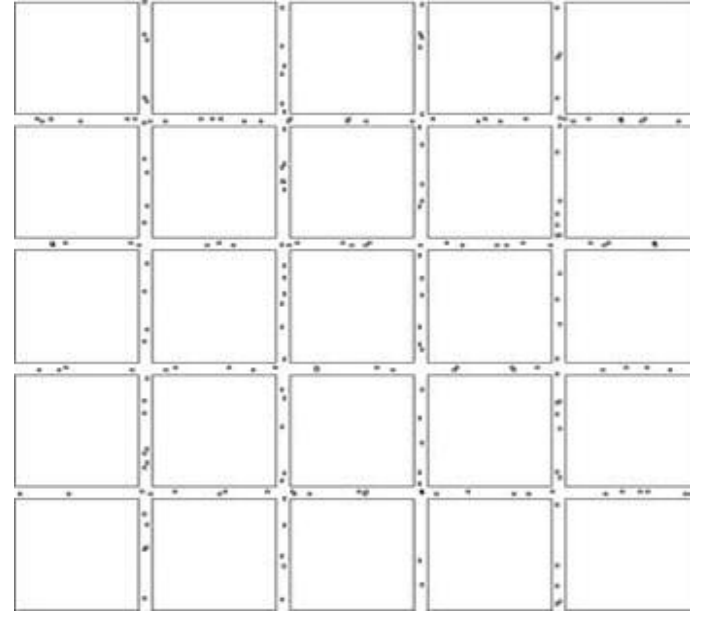

Fig 2: Manhattan Mobility Model

To reflect better the vehicular movement, interactions among vehicles as well as between vehicles and the environment are described by flow models [9]. Flow models specify rules for car following, lane changing and overtaking behavior and are categorized by the level of granularity as: macroscopic, mesoscopic and microscopic. Macroscopic models represent traffic as fluid streams by using aggregated values such as density or average velocity. Mesoscopic models describe some interactions among cars at an individual level, but they base the interactions on general characteristics. Microscopic models consider mobility of each individual vehicle. They implement realistic car following behavior by adjusting each vehicle's velocity in respect to the vehicle in front (e.g. Intelligent Driver Model [10], Krauss Model [11]). For VANET simulations, where communication among individual vehicles needs to be available, microscopic models are the most suitable.

\section{PROPAGATION MODELS}

The propagation model is defined as a mechanism to estimate the Received Signal Strength (RSS) of wireless transmission [12]. Factors that are used to estimate the RSS include transmission power, distance between sender and the receiver, height of antenna etc. Once this RSS is calculated, it is then used to determine whether a packet is received successfully or not [13] [14].

Radio propagation is the behavior of radio waves when they are transmitted, or propagated from one point on the Earth to another, or into various parts of the atmosphere [15]. Like light waves, radio waves are affected by the phenomena of reflection, refraction, diffraction, absorption, polarization and scattering.

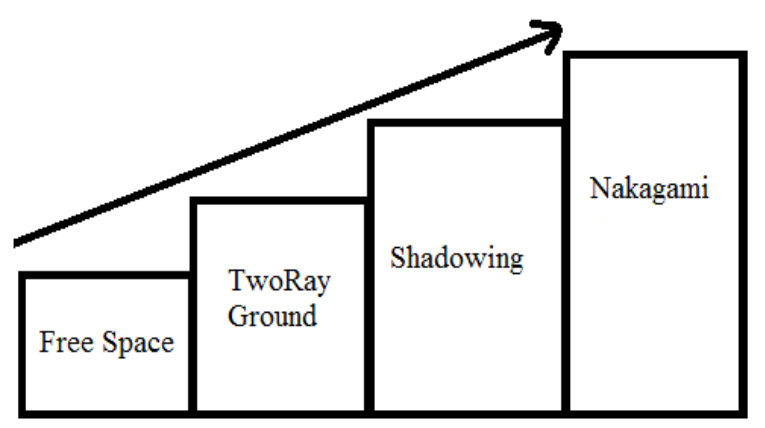

Fig. 3: Radio Propagation Models Evolution for VANETs
Fig. 3 represents the radio propagation models used and the evolution of it from free space to Nakagami radio propagation for fading scenario. This section elaborates the details of each radio propagation models.

\subsection{Free Space}

This radio propagation model is earliest model and was used in MANET by researchers in the earliest studies. In Free Space model, signal propagating through open space, with no environmental effects. It has one parameter, called "line-ofsight". If there is no LOS, the signal is blocked entirely and no communication takes place [16].

\subsection{TwoRayGround}

This radio propagation model is highly preferred in MANET by researchers. This radio propagation model is also used in maximum research studies for protocols performances in VANET scenario. It has been observed that the TwoRayGround model is a more realistic than the Free-Space model as it addresses the case while considering a ground reflected propagation path between transmitter and receiver, in addition to the direct LOS path.

This model is especially useful for predicting the received power at large distances from the transmitter and when the transmitter is relatively high above the ground. It is interesting to note that at far distances, the received power becomes independent of the frequency [17].

\subsection{Shadowing}

This model has many possible scenarios that can affect radio signal power. The shadowing model defines the path loss model, which also predicts the mean received power at distance [18].

\subsection{Nakagami}

Nakagami [19] is a mathematical general modeling of a radio channel with fading. With help of comparative study, found that rather than to existing models, Nakagami model has more configurable parameters. To allow a closer representation of the wireless communication channel, and it is the perfect for free space channel.

\section{ROUTING PROTOCOLS}

In wireless ad-hoc networks, routing protocols are used to calculate efficient routes. These protocols are divided into two main categories with respect to their routing behavior; on demand (reactive) and table driven (proactive) [13]

In this section these two classes of routing protocols are defined. Both these protocols have evolved over the years and have been used extensively in simulation based study.

Proactive routing protocols continuously update the routing table, even when there is no communication going on. This makes these protocols to generate sustained routing overhead, but the benefit is that no latency of route discovery process is involved. Routes to the destinations are readily available and can be used as soon as application has data to send. It is based on periodic exchange of control messages and maintaining routing tables, that is why these are known as table-driven routing protocols for complete implementation of topology locally.

Reactive routing protocols incur initial route discovery latency by using flooding to discover routes, which is their main overhead. These routing protocols calculate routes for destination in the network, when it is needed therefore these 
are known as on-demand routing protocols. Applications have to wait for the routes to be discovered and then use them for communication.

Unlike proactive routing protocols, reactive protocols do not generate sustained routing overhead. Reactive protocols usually take more time to find a route as compared to a proactive protocol [20]. Both these approaches have their pros and cons and no single routing protocol is able to work efficiently in all possible scenarios. Ad-hoc On demand Distance Vector (AODV) protocol [21], DSR [22] and DYMO [23] belongs to reactive routing protocol family, while DSDV [24][25] and Optimized Link State Routing (OLSR) protocol [25] is an example of proactive routing protocols [26][27][28][29].

Destination sequenced distance vector routing (DSDV) is adapted from the conventional Routing Information Protocol (RIP) to ad-hoc networks routing. It was design by C. Perkins and P. Bhagwat. The main contribution of the algorithm is useful to solve the routing loop problem. Each entry in the routing table consist of a sequence number, the sequence numbers are set even if a link is present; else, an odd number is denote. From generated destination number, the emitter needs to send out the next update with this number. Routing information is spread between nodes by sending full dumps infrequently and smaller incremental updates more frequently [30].

The Optimized Link State Routing Protocol (OLSR) is developed for mobile ad-hoc networks. It operates as a table driven, proactive protocol, i.e., exchanges topology information with other nodes of the network regularly. Each node selects a set of its neighbor nodes as "multipoint relays" (MPR). In OLSR, only nodes, selected as such MPRs, are responsible for forwarding control traffic, intended for diffusion into the entire network. MPRs provide an efficient mechanism for flooding control traffic by reducing the number of transmissions required [25].

AODV [21] is a distance vector routing protocol when a node wants to establish new communication with another node, it searches for an available path to the destination node, in its local routing table. If there is no availability of path, then it sends a route request (RREQ) message to its neighborhood. The nodes that receive this message search for a path leading to the destination node. If there is no path then, it re-send the RREQ message and establish a path leading to RREQ originating node.

Ad-hoc On-demand Multipath Distance Vector Routing (AOMDV) protocol is a modification of the AODV protocol for computing multiple loop-free and link disjoint paths. The routing entries for each destination consist of a list of the next-hops along with the respective hop counts. All the next hops have the same sequence number.

\section{ANALYSIS}

The analysis of Mobility Models, Propagation Model and Routing Protocols was performed to find the best among themselves for the Indian automotive networks scenario. In this each sub section provides the detailed analysis of each of them.

\subsection{Mobility Models}

In order to produce realistic mobility patterns for the Indian automotive networks, proper vehicular mobility models must therefore be defined.
Stochastic mobility models, like Random Way-Point or models with constrained movement (Manhattan) are not able to reproduce the realistic traffic mobility [8]. To reflect the better vehicular movement, interactions among vehicles as well as between vehicles and the environment are described by flow models [9]. The three classes of mobility flow models are analyzed in the below mentioned sub section.

\subsubsection{Microscopic Mobility}

- It simulate the movement of every single vehicle on the street, mostly assuming that the behavior of the vehicle depends on both, the vehicle's physical abilities to move and the driver's controlling behavior.

- Traffic flow describes the mobility parameters of a specific car with respect to other cars in detail.

- Enhanced with lane-changing models.

- An intersection with traffic lights or signs is modeled similarly to a stopped vehicle. The driver model acquires the state of the traffic sign/light and moves accordingly.

\subsubsection{Macroscopic Mobility}

- It does not consider the mobility parameters of a specific car but instead quantities of macroscopic meanings such as flow, speed, or density are modeled.

- The macroscopic approach is inspired by hydrodynamic phenomena and do not intend to reproduce individual but rather macroscopic quantities.

- This models cannot model individual vehicles, stop or yield signs and complex traffic light patterns are difficult to model [31] [32].

\subsubsection{Mesoscopic Mobility}

- It is located at the boundary between microscopic and macroscopic simulations.

- Vehicle movement is mostly simulated using queue approaches and single vehicles are moved between such queues.

- The objective is to benefit from the scalability of the macroscopic approach but still providing a detailed modeling close to microscopic models.

- Represent an intermediate modeling level of traffic flows and model gross characteristics of traffic flows at an aggregate level, typically as probability density functions, but describe the interactions between vehicles at an individual level [33-35].

\subsection{Propagation Models}

The various propagation models are analyzed in the below mentioned sub section.

\subsubsection{Free Space}

This propagation model is not suitable for VANET. The received power is only dependent on the transmitted power, the antenna gains and on the distance between the sender and the receiver. Obstacles are not modeled. In VANET, vehicles have to move in city where obstacles are main concerns as the 
buildings alongside the road will block the transmission or communication.

\subsubsection{TwoRayGround}

This model does not give a good result for a short distance due to the oscillation caused by the constructive and destructive combination of the two rays. This model is not perfect for VANET.

\subsubsection{Shadowing}

The shadowing radio propagation model can be used in VANET scenario by varying either $\beta$ values to set the various environment conditions for obstacles.

\subsubsection{Nakagami}

According to simulation results, this model can be suitable from a perfect free space channel, to a moderate fading channel on highway, even to a dramatically fading channel in urban communities. By varying shape factor $\mathrm{m}$ value, form the highly fading scenarios like urban or city as well as the free way of highway. This radio propagation model is highly applicable for the use in protocol performances for VANETs.

\subsection{Routing Protocols}

The In this sub section the performance of various routing protocols is analyzed for the Indian automotive network. The major objective is to find the suitable routing protocol for the Indian intelligent transport network. The performance analysis of various routing protocols such as AODV, DSDV, DSR, OLSR routing protocols depends on various parameters such as Throughput, Packet delivery ratio and Delay etc.

The author of paper [36] analyzed the AODV, DSDV and DSR routing protocols for wireless networks by calculating the throughput and packet loss percentage. They concluded that AODV has highest throughput and best suited for large network. The same protocols are compared on the basis of packet size in paper [37]. This shows that DSR is more efficient as compared to AODV and other routing protocols.

In the paper [38] [39] author evaluated the performance of AODV, AOMDV DSR, OLSR and DSDV routing protocols on the basis of Packet delivery fraction and Delay percentage. Author found that AODV shows better result as compared to other routing protocols. When these protocols compared on the basis of cluster size in paper [40], author concluded that DSR is best suited for small cluster, whereas AODV is more suitable for large clusters.

The performance of AODV, DSDV and OLSR is evaluated on the basis of length, acceleration and max speed. It shows that AODV provides the best communication link during information exchange [41].Authors of paper [42] compared different VANET routing protocols on different parameters. They concluded that overall performance of AODV with IEEE $802.11 \mathrm{p}$ is better as compared to DSDV. Table 1 provides the summary of various routing protocols.

Table 1. Performance analysis of routing protocols for VANET

\begin{tabular}{|c|c|c|c|c|}
\hline $\begin{array}{c}\text { Criteria / } \\
\text { Protocols }\end{array}$ & AODV & DSR & DSDV & OLSR \\
\hline Throughput & High & Average & Average & Low \\
\hline Packet Size & Average & High & Average & Average \\
\hline PDF & High & Average & Average & Average \\
\hline
\end{tabular}

\begin{tabular}{|c|c|c|c|c|}
\hline $\begin{array}{c}\text { Criteria / } \\
\text { Protocols }\end{array}$ & AODV & DSR & DSDV & OLSR \\
\hline Delay $\%$ & Average & Average & Average & Average \\
\hline Cluster Size & High & Average & Low & Low \\
\hline Overhead & Low & Average & High & Average \\
\hline $\begin{array}{c}\text { Traffic } \\
\text { Load }\end{array}$ & High & Average & Average & Average \\
\hline
\end{tabular}

Table 1 analyzed that AODV and DSR are more suitable for VANET research. At the same time AODV considered for Indian networks, as it has high throughput and load overhead. It also supports high cluster area.

\section{CONCLUSION}

In this paper the performance of various Models and Routing Protocols for Intelligent Transport System is evaluated. They are considered for Indian urban and rural scenario. In order to produce realistic mobility patterns for the Indian automotive networks, microscopic vehicular mobility models is more suitable as compare to macroscopic, mesoscopic models. Nakagami propagation model is more suitable for different scenarios like urban or city as well as the free way of highway. This radio propagation model is highly applicable for the use in protocol performances in VANETs. Finally AODV and DSR are more suitable for VANET research. At the same time AODV was considered for Indian networks, as it has high throughput and load overhead. It also supports high cluster area. They use VANET's IEEE 802.11 standards and wireless channels for realistic simulation. This analysis helps us in performing the realistic evaluation of the intelligent transport system for the Indian automotive networks.

\section{ACKNOWLEDGMENTS}

I would like to thanks all mighty God and my Babu Jee for his kind blessing. I want to express my sincere regards to my family and friends for their unconditional support throughout my life. Thank you every one for your love and support.

\section{REFERENCES}

[1] Muhammad Alam, et al., "Integrated Mobility Model (IMM) for VANETs Simulation and its Impact", International Conference on Emerging Technologies, 2009.

[2] V. S. Dhaka, et al., "The Realistic Mobility Evaluation of Vehicular Ad-Hoc Network for Indian Automotive Networks", Published at International Journal of Ad hoc, Sensor \& Ubiquitous Computing (IJASUC), Vol. 5, No.2, April 2014, ISSN: 0976-1764 [Online]; 0976-2205 [Print].

[3] Aslinda Hassan, et al., "Performance Evaluation for Multicast Transmissions in VANET".

[4] Trupti G.Nimje \& S. S. Dorle, "A Survey on Various Mobility Models to improve Realistic Simulation and Accuracy of IVC Protocols", IEEE International Conference on Emerging Trends in Computing, Communication and Nanotechnology (ICECCN 2013), 2013.

[5] James C. Williams, "Macroscopic Flow Models".

[6] M. Fiore and J. Harri, "The Networking Shape of Vehicular Mobility", Proc. of the 9th ACM International 
Symposium on Mobile Ad Hoc Networking and Computing, 2008, pp. (s): 261-272, ISBN: 978-1-60558073-9, doi: 10.1145/1374618.1374654.

[7] J. Harri et al., "Mobility models for vehicular ad hoc networks: A survey and taxonomy", In Communications Surveys \& Tutorials, IEEE, Dec 2009, Vol. 11, no. 4, pp. (s): 19-41, ISSN: 1553-877X, doi: 10.1109/SURV.2009 .090403 .

[8] R. Baumann et al., "Generic mobility simulation framework (gmsf)", Proc. of the 1st ACM SIGMOBILE Workshop on Mobility Models, May 2008, pp. (s): 4956, ISBN: 978-1-60558-111-8, doi: 10.1145/1374688. 1374698.

[9] H. Hartenstein and K. Laberteaux, "VANET: vehicular applications and inter-networking technologies", Wiley Online Library, 2010.

[10] M. Treiber et al., "Congested traffic states in empirical observations and microscopic simulations", Physical Review E, 2000, Vol. 62, no. 2, pp.: 1805.

[11] S. Krauss et al., "Metastable states in a microscopic model of traffic flow", Physical Review E, 1997, Vol. 55 , no. 5 , pp.: 5597.

[12] S. Krauss, "Microscopic Modeling of Traffic Flow: Investigation of Collision Free Vehicle Dynamics", PhD thesis, Universitat zu Koln, 1998.

[13] I. Khan and A. Qayyum, "Performance Evaluation of AODV and OLSR in Highly Fading Vehicular Ad hoc Network Environments", In 13th IEEE International Multitopic Conference, Dec. 2009, pp. (s): 1 - 5, ISBN: 978-1-4244-4872-2, doi: 10.1109/INMIC.2009.5383121.

[14] M. M. Torrent et al., "Effects of a Realistic Channel Model On Packet Forwarding in Vehicular Ad Hoc Networks", Proc. of the IEEE Wireless Communications and Networking Conference (WCNC), April, 2006, Las Vegas, Nevada, Vol. 1, pp. (s): 385-391, ISSN: 15253511, ISBN: 1-4244-0269-7, doi: 10.1109/WCNC.2006. 1683495 .

[15] P. K. Singh, K. Lego, "Comparative Study of Radio Propagation and Mobility Models in Vehicular Ad-hoc Network", In International Journal of Computer Application, Feb. 2011, Vol. 16, no. 8, pp. (s): 37-42, ISSN: 0975 - 8887.

[16] M. Siraj \& S. Kanrar, "Performance of Modeling wireless networks in Realistic environment" In International Journal of Computer Networks (IJCN), 2010, Vol. 2, no. 1, pp. (s): 62-79, ISSN: 1985-4129.

[17] M. Khosroshahy et al., "Snapshot of MAC, PHY and Propagation Models for IEEE 802.11", In Open-Source Network Simulators", Project Report, INRIA, Sep. 2007.

[18] I. K. Eltahir, "The impact of different radio propagation models for Mobile Ad-hoc NETworks (MANET) in urban area environment", In IEEE 2nd International Conference on Wireless Broadband and Ultra Wideband Communications (AusWireless), Sydney, NSW, Aug. 2007, pp.: 30, ISBN: 978-0-7695-2846-5, doi: 10.1109/AUSWIRELESS.2007.80.

[19] F. J. Martinez et al., "Realistic Radio Propagation Models (RPMs) for VANET Simulations", In IEEE
Wireless Communications and Networking Conference (WCNC), April 2009, pp. (s): 1-6, ISSN: 1525-3511, ISBN: 978-1-4244-2947-9, doi: 10.1109/WCNC.2009 .4917932.

[20] S. Sagar, et al., "Analysis and Modeling Experiment Performance Parameters of Routing Protocols in MANETs and VANETs", in IEEE 11th International Conference on Trust, Security and Privacy in Computing and Communications, Liverpool, June 2012, pp. (s): 1867 - 1871, ISBN: 978-1-4673-2172-3, doi: 10.1109/ TrustCom.2012.89.

[21] C. Perkins, et al. "Ad Hoc On - Demand Distance Vector (AODV) Routing”, RFC 3561, Network Working Group, 2003.

[22] D. Johnson et al., "The Dynamic Source Routing Protocol (DSR) for Mobile Ad Hoc Networks for IPv4", In Network Working Group, RFC 4728, Feb. 2007.

[23] I. Chakeres and C. Perkins, "Dynamic MANET Ondemand (DYMO) Routing", IETF Internet Draft, draftietf-manet-dymo-12.txt, 2008.

[24] C. E. Perkins and P. Bhagwat, "Highly dynamic Destination-Sequenced Distance- Vector routing (DSDV) for Mobile Computers", Proc. of the ACM Conference on Communications architectures, protocols and applications, NY, USA, Oct. 1994, Vol. 24, no. 4, pp. (s): 234-244, ISBN: 0-89791-682-4, doi: 10.1145/ 190314.190336.

[25] T. Clausen et al., "Optimized Link State Routing Protocol (OLSR)", In Network Working Group, RFC 3626, Oct. 2003.

[26] M. Azouqa et al., "Investigation of Vehicle-To-Vehicle Communication In A Road Intersection", At Department of Electronic Engineering Al-Quds University, Jerusalem, Palestine, ISBN: 978-9957-469-01-6.

[27] A. Shastri, et al., "Performance Analysis of On-demand Routing Protocols for Vehicular Ad-hoc Networks", In International Journal of Wireless and Mobile Networks (IJWMN), Aug. 2011, Vol. 3, no. 4, pp. (s): 103-111, ISSN: 0975-4679.

[28] R. Singh, et al., "Performance Evaluation of DSR and DSDV Routing Protocols for Wireless Ad Hoc Networks", In International Journal of Advanced Networking and Applications, 2011, Vol. 02, no. 4, pp. (s): 732-737, ISSN: 0975-0282.

[29] A. Kumar and A. K. Singla, "Performance evaluation of MANET Routing Protocols on the basis of TCP Traffic Pattern", In International Journal of Information Technology Convergence and Services (IJITCS), Oct. 2011, Vol. 1, no. 5, pp. (s): 41-48, ISSN: 2231-1939.

[30] G. He, "Destination-Sequenced Distance Vector (DSDV) Protocol", At Networking Laboratory, Helsinki University of Technology, 2002.

[31] S. P. Hoogendoorn and PHL Bovy, "State-of-the-art of Vehicular Traffic Flow Modelling", In Journal of Systems and Control Engineering - Special Issue on Road Traffic Modeling and Control, June 2001, Vol. 215, no. 4, pp. (s): 283-303, doi: 10.1177/09596518012 1500402 . 
[32] K. Nagel, "Still Flowing: Approaches to Traffic Flow and Traffic Jam Modeling", In Operations Research, 2003, Vol. 51, no. 5, pp. (s): 681-710, doi: 10.1287/opre.51.5.681.16755.

[33] I. Prigogine and R. Herman, "Kinetic Theory of Vehicular Traffic", In Workshop Arizona State University, Elsevier, NewYork, 2003.

[34] C. Gawron, "An Iterative Algorithm to Determine the Dynamic User Equilibrium in a Traffic Simulation Model”, In International Journal of Modern Physics C, 1998, Vol. 9, no. 3, pp. (s): 393-407, ISSN: 0129-1831, doi: 10.1142/S0129183198000303.

[35] N. Cetin et al., "A large-scale Multi-agent Traffic Microsimulation based on Queue Model", In Swiss Transport Research Conference (STRC), 2003.

[36] S. B. Monika and A. Dhiman, "Comparative Study of AODV, DSDV and DSR Routing Protocols in Vehicular Network Using EstiNet Simulator," International Journal of Scientific \& Engineering Research, Vol. 3, 2012.

[37] S. M. Feiz and A. Movaghar, "Comparison of DSR, AODV and DSDV Routing Protocols in Fully and Partially Connected VANET," Proceedings of 2011 World Congress in Computer Science, Computer Engineering and Applied Computing, Las Vegas, Nevada, July $18-21,2011$.
[38] S. S. Dorle, et al., "Evaluation of Multipath, Unipath and Hybrid Routing Protocols for Vehicular Ad Hoc Networks," in Emerging Trends in Engineering and Technology (ICETET), 4th International Conference, 2011, pp. (s): 311-316.

[39] M. U. Nagaraj and M. P. Dhamal, "Performance Comparison of AODV, AOMDV, OLSR, DSR and GSR Routing Protocols in VANET," UACEE International Journal of Advances in Computer Networks and its Security, Vol. 2, 2012.

[40] Y. M. Sharma and S. Mukherjee, "Comparative Performance Exploration of AODV, DSDV \& DSR routing protocols in Cluster Based VANET Environment," International Journal of Advances in Engineering \& Technology (IJAET), Vol. 4, 2012.

[41] G. Z. Santoso and M. Kang, "Performance analysis of AODV, DSDV and OLSR in a VANETs safety application scenario," in Advanced Communication Technology (ICACT), 2012 14th International Conference, 2012, pp. (s): 57-60.

[42] A. N. Mahajan, et al., "Analysis and Comparison of VANET Routing Protocols using IEEE 802.11p and WAVE", International Journal of Engineering Research and Applications (IJERA), Vol. 3, Issue 4, Jul-Aug 2013, pp. (s): 1556-1561, ISSN: 2248-9622.owman, M., Debray, S. K., and Peterson, L. L. 1993. Reasoning about naming systems. 